\title{
Efficacy of Shankhapushpi Choorna on Borderline IQ in School Going Children - A Randomized Placebo Controlled Clinical Study
}

\author{
Research Article
}

Aswathi P Murali1 ${ }^{*}$, Prathviraj Puranik², Nagaratna S Jartarghar ${ }^{3}$

1. PG Scholar, 2. HOD \& Professor, 3. Assistant Professor

Department of PG Studies in Kaumarabhritya,

Sri Dharmasthala Manjunatheshwara College of Ayurveda and Hospital, Udupi, KA, India.

\begin{abstract}
Objective: To study the concepts regarding Medha, Medhya and IQ. Detailed assessment of IQ level of school going children with 6-8 years of age group. To study the efficacy of Shankhapushpi Choorna in the enhancement of IQ of school going children with Borderline IQ. Design: Open randomized placebo controlled clinical study with pre and post-test design. Setting: O.P.D. of Sri Dharmasthala Manjunatheshwara College of Ayurveda and Hospital, Udupi and also nearby schools of Udupi. Interventions: A minimum of 40 children under borderline IQ were selected based on the inclusion and exclusion criteria. The selected children were randomly divided into 2 groups of 20 each. Group A (Trial Group) were treated with Shankhapushpi Choorna with dose of 5 grams daily at night, after food with lukewarm milk for a duration of 60 days and Group B was administered with Placebo. The follow up period was 30 days after the intervention. Main outcome measures: The subjective criteria were based on the parameters of Grasping power, Memory power, Courage \& Activity. The values obtained using Malin's Intelligence Scale for Indian Children (MISIC) were considered for objective assessment. Results: Over the duration of the study, the trial drug Shankhapushpi Choorna was seen to have a positive effect on all the subjective and objective parameters with statistically highly significant results. Conclusion: Shankhapushpi Choorna with the proper dosage according to the age definitely improves the Medha of children with borderline IQ. The drug can be used as both promotive as well as curative aspects in accordance with intelligence.
\end{abstract}

Key Words: Medha, Medhya Rasayana, Intelligence, Intelligence Quotient, Convolvulus pluricaulis Choisy, MISIC.

\section{Introduction}

Kaumarabhritya is one of the important branches of Ashtanga Ayurveda which concerns with the complete wellbeing of children. A child is advised to be brought up from childhood to adolescence in accordance with principles of Ayurveda for attaining the ultimate aim of Purusharthas (1). Health is the integral state of normalcy of both Sharira as well as Manas. The functions attributed to the Manas forms the basic foundation for the phenomena of knowledge hence the dynamicity of Manas controls the process of cognition.

The present era eager to analyse the word Intelligence, since it has a definite impact on many human behaviours. It is having a multitude of definitions like the capacity for logic, understanding, self-awareness, learning, emotional knowledge, reasoning, planning, creativity, critical thinking and problem solving (2). IQ (the Intelligence Quotient) is the quantification of an individual's intelligence power

\section{* Corresponding Author:}

\section{Aswathi P Murali}

Department of PG Studies in Kaumarabhritya,

Sri Dharmasthala Manjunatheshwara College of

Ayurveda and Hospital,

Udupi, KA, India

Email Id: aswathipmurali@gmail.com relative to peers of a similar age by using different assessment tools. The individual's score on a modern IQ test is a good predictor of many life outcomes, including educational and career success (3).

Childhood is the timespan where the immature dhatus sustains the body and undergoes maximum growth and development (4). This is the apt stage for diagnosing mental vulnerabilities that most of them can make the future challenging. Definitely convenient early interventions are needed to boost the intellectual growth in children. Good mental health abides the platform for skilful development in children. A child with depreciated learning capacity does not adjust well socially (5). Mental inability is a crucial public hazard and World Health Organization estimates that $10 \%$ of the world's population has some form of mental disability and many of them are correctable if detected early.

Borderline IQ or borderline intellectual functioning is a categorization of intelligence of a person who has below average cognitive ability but the deficit is not as severe as intellectual disability. This is technically a cognitive impairment; however, this group may not be sufficiently mentally disabled but they may need some specialized care. In school age group, children with borderline IQ are often slow learners (6).

Medha can be understood as the faculty of Buddhi which has the power to retain the personal experiences and when needed it recalls that retained 
knowledge thus it prevents an individual from indulging into Prajnaparadha (7). The faculty of Medha can be understood as in two ways; Grahanashakti (Grasping Power) and Dharanashakti (Retention Power) (8). The textbook of Amarakosha explained Medha as the faculty needed to have proper correlation and understanding about the knowledge of the existing objects. The knowledge cannot be realized in the absence of Medha (9). In general, Medha is the power of intelligence that restores the knowledge and this restoration leads to improve the individual database of different knowledge.

Ayurveda provides the evidence-based quantum of pharmacological knowledge for the improvement of Medha and Buddhi and this is the exceeding time limit for the analysis as well as the rightful utilization of such valuable drugs for the needy ones. Medhya Rasayana drugs are those which improves mental faculties and intellectual functions (10). Among the 'Chaturvidha Medhya Rasayana' (Group of four Medhya drugs), it has been told that 'Medhya viseshena cha Shankhapushpi', the foremost and excellent drug is Shankhapushpi among them having the potency to improve intellectual functions (11).

Shankhapushpi is a well-known herb since the most primitive authentic works on Ayurveda have its therapeutic utility well elaborated and its morphological characters are well described. Later on, controversies flare up as local community in different parts of India used different plant species in the name of Shankhapushpi. Ayurvedic Pharmacopoeia of India has established that Convolvulus pluricaulis is the plant species which should be taken in the name of Shankhapushpi and thus put an end to the controversial status of the plant moreover (12).

Our classics enlighten the promotion of Medha, Buddhi and Smruti with several formulations mentioned which impacts great potential to improve the mental power and intelligence. The effect of Ayurvedic drugs in promoting intellectual capacity should be subjected for a thorough analysis is the need of the day. Even though various pharmacological and experimental studies have proven the versatile action of Shankhapushpi as medhya drug, no documental efficacy studies are conducted on children for improvement of borderline IQ. Hence Shankhapushpi (Convolvulus pluricaulis Choisy) was selected as trial drug in the present study.

\section{Materials and Methods}

- To study the efficacy of Shankhapushpi Choorna in the enhancement of IQ of school going children with Borderline IQ.

- Study design: A randomized placebo controlled clinical study with pre-test and post-test design.

- Source of data: 40 Children of age group 6-8 years were selected from Kaumarabhritya OPD of SDM College of Ayurveda \& Hospital, Udupi and also nearby schools of Udupi, Karnataka.

- Method of collection of data: 40 children were randomly divided into 2 groups, Group A (Trial Group) and Group B (Placebo controlled) with 20 children each.

\section{Inclusion criteria}

Students in the age group of 6-8 years irrespective of sex, caste, religion, socio-economic status having IQ of 70 to 89 (Borderline IQ).

\section{Exclusion criteria}

- Children below the age of 6 years and above the age of 8 years.

- Children having IQ less than 70 and more than 89.

- Children with Neurobehavioral disorders like ADHD, Autism and Intellectual Disability.

- Children suffering from any chronic illness, systemic disorders and on treatment.

- Children with Congenital abnormalities, Genetic disorders.

- Children with Psychiatric illness and Personality disorders.

\section{Diagnostic Criteria}

The assessment of the effect of the drug under trial was done based upon the subjective and objective criteria.

\section{Subjective Parameters \\ - Grasping power \\ - Memory power \\ - Courage and Activity.}

\section{Objective Parameters}

The values obtained using "Malin's Intelligence Scale for Indian Children" (MISIC) were considered for objective assessment. The test comprises of 11 subtests divided into two groups, Verbal and Performance.

\section{Intervention}

- Drug: Shankhapushpi (Convolvulus pluricaulis choisy) Choorna

- Drug Source: GMP certified S D M Pharmacy, Udupi

- Part Used: Whole plant

\section{Collection \& Authentication of Raw Drug}

The raw drug Shankhapushpi in dry form was collected from the SDM Pharmacy of Ayurveda, Udupi, Karnataka state, India. The drug analysis and standardization were done at SDM Centre for Research in Ayurveda and Allied Sciences, Udupi, Karnataka state, India.

\section{Administration Method}

- Group A: Group A contained 20 children who received Shankhapushpi Choorna with dose of 5 grams daily at night, after food with lukewarm milk for a duration of 60 days.

- Group B: Group B contained 20 children who received Placebo (Starch powder) with dose of 5 grams daily at night, after food with lukewarm milk for a duration of 60 days.

\section{Duration of the study}

Total duration of the study: 90 days

Duration of intervention: 60 days

Follow up: 30 days 
Period of Assessment

The children will be assessed on 0th day, 30th day, 60th day and on 90th day for follow up.

Statistical Evaluation

The data was coded and entered into Microsoft Excel Spreadsheet. Analysis was done using Statistical Package for Social Science (SPSS) version 20 (SPSS Inc. Chicago, IL, USA) Windows software program.

\section{Results}

Effect of treatment on Subjective Parameters

Statistical Analysis within the Group was analysed by using Wilcoxon signed - rank test and in between Group A and Group B by Mann - Whitney U test.

Group A showed highly significant improvement on Grasping Power \& Memory Power, before treatment and after treatment \& before treatment and on follow up. Group B showed non-significant result on every level of assessment. Group A showed significant improvement on Courage \& Activity, before treatment and after treatment \& before treatment and on follow up. Group B showed non-significant result on every level of assessment.

Table No.1: Results of Shankhapushpi Choorna for the parameter Grasping Power

\begin{tabular}{|c|c|c|c|c|c|c|c|c|c|c|c|}
\hline \multicolumn{4}{|c|}{$\begin{array}{c}\text { BT Mean } \\
2.25\end{array}$} & \multicolumn{3}{|c|}{$\begin{array}{c}3^{\text {th }} \text { day Mean } \\
2.10\end{array}$} & \multicolumn{3}{|c|}{$6^{\text {th }}$ day Mean } & \multicolumn{2}{|c|}{$9^{\text {th }}$ day Mean } \\
\hline \multicolumn{12}{|c|}{ Grasping Power - GROUP A } \\
\hline \multirow{2}{*}{ Grasping power } & \multicolumn{3}{|c|}{ Negative Ranks } & \multicolumn{3}{|c|}{ Positive Ranks } & \multirow{2}{*}{ Ties } & \multirow{2}{*}{ Total } & \multirow{2}{*}{$Z$ value } & \multirow{2}{*}{ P value } & \multirow{2}{*}{$\begin{array}{c}\text { Inter- } \\
\text { pretation }\end{array}$} \\
\hline & $\mathbf{N}$ & MR & SR & $\mathbf{N}$ & MR & SR & & & & & \\
\hline BT-30 th day & 3 & 2 & 6 & 0 & 0.00 & 0.00 & 17 & 20 & -1.73 & 0.083 & NS \\
\hline BT-60 th day & 13 & 7 & 91 & 0 & 0.00 & 0.00 & 7 & 20 & -3.50 & 0.000 & HS \\
\hline $60^{\text {th }}$ day- $-90^{\text {th }}$ day & 5 & 3 & 15 & 0 & 0.00 & 0.00 & 15 & 20 & -2.23 & 0.025 & $\mathrm{~S}$ \\
\hline BT-90 ${ }^{\text {th }}$ day & 16 & 8.5 & 136 & 0 & 0.00 & 0.00 & 4 & 20 & -3.75 & 0.000 & HS \\
\hline
\end{tabular}
N - Sample size $\quad$ MR - Mean Rank $\quad$ SR - Sum of Ranks

Table No.2: Results of Placebo for the parameter Grasping Power

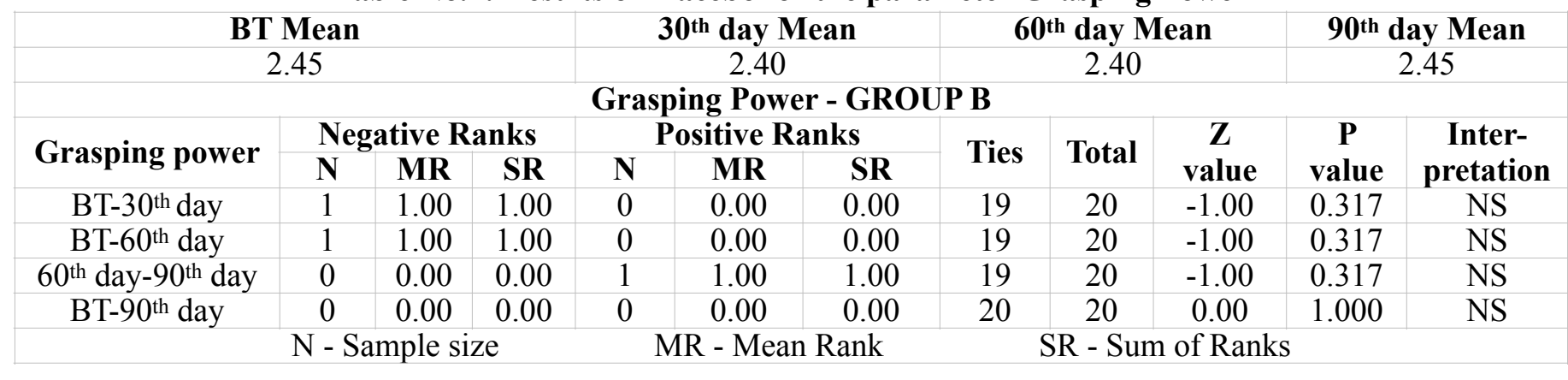

Table No.3: Results of Shankhapushpi Choorna for the parameter Memory Power

\begin{tabular}{|c|c|c|c|}
\hline BT Mean & $3^{\text {th }}$ day Mean & $\mathbf{6 0}^{\text {th }}$ day Mean & $\mathbf{9 0}^{\text {th }}$ day Mean \\
\hline 2.75 & 2.60 & 1.95 & 1.75 \\
\hline
\end{tabular}

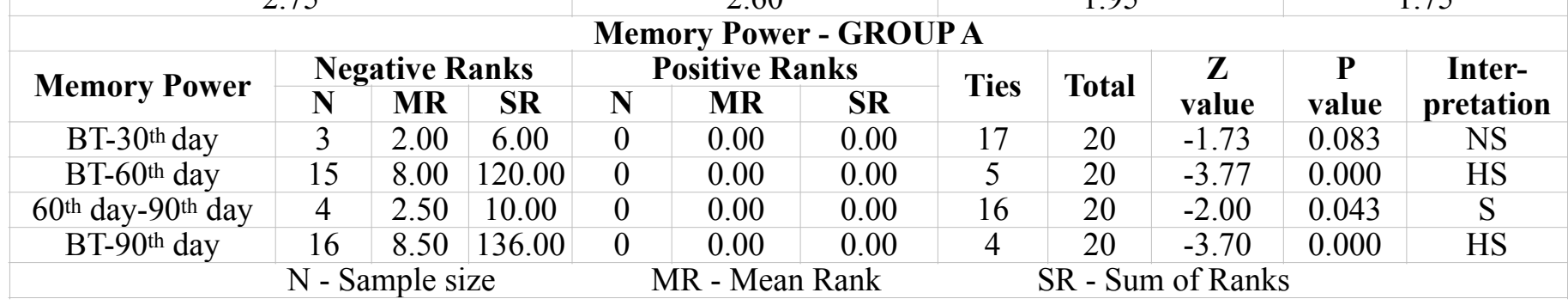

Table no.4: Results of Placebo for the parameter Memory Power

\begin{tabular}{|c|c|c|c|}
\hline BT Mean & $\mathbf{3 0}^{\text {th }}$ day Mean & $\mathbf{6 0}^{\text {th }}$ day Mean & $\mathbf{9 0}^{\text {th }}$ day Mean \\
\hline 2.90 & 2.85 & 2.80 & 2.80 \\
\hline
\end{tabular}

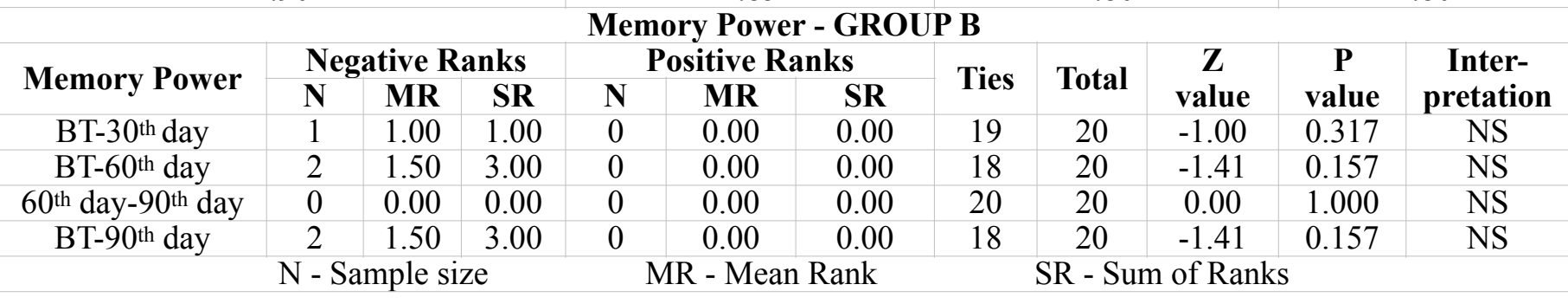


Aswathi P Murali et.al., Efficacy of Shankhapushpi Choorna on Borderline IQ in School Going Children

Table no.5: Results of Shankhapushpi Choorna for the parameter Courage \& Activity

BT Mean

0.45 $30^{\text {th }}$ day Mean

0.35 $6^{\text {th }}$ day Mean

0.10 $90^{\text {th }}$ day Mean

Courage \& Activity - GROUP A

\begin{tabular}{|c|c|c|c|c|c|c|c|c|c|c|c|}
\hline \multirow{2}{*}{$\begin{array}{c}\text { Courage \& } \\
\text { Activity }\end{array}$} & \multicolumn{3}{|c|}{ Negative Ranks } & \multicolumn{3}{|c|}{ Positive Ranks } & \multirow{2}{*}{ Ties } & \multirow{2}{*}{ Total } & \multirow{2}{*}{$\begin{array}{c}Z \\
\text { value }\end{array}$} & \multirow{2}{*}{$\begin{array}{c}P \\
\text { value }\end{array}$} & \multirow{2}{*}{$\begin{array}{c}\text { Inter- } \\
\text { pretation }\end{array}$} \\
\hline & $\mathbf{N}$ & MR & SR & $\mathbf{N}$ & MR & SR & & & & & \\
\hline BT-30"th day & 2 & 1.50 & 3.00 & 0 & 0.00 & 0.00 & 18 & 20 & -1.41 & 0.157 & NS \\
\hline BT-60th day & 7 & 4.00 & 28.00 & 0 & 0.00 & 0.00 & 13 & 20 & -2.64 & 0.008 & $\mathrm{~S}$ \\
\hline $0^{\text {th }}$ day-90 $90^{\text {th }}$ day & 0 & 0.00 & 0.00 & 0 & 0.00 & 0.00 & 20 & 20 & 0.00 & 1.000 & NS \\
\hline BT-90th day & 7 & 4.00 & 28.00 & 0 & 0.00 & 0.00 & 13 & 20 & -2.64 & 0.008 & $\mathrm{~S}$ \\
\hline
\end{tabular}

Table no.6: Results of Placebo for the parameter Courage \& Activity

BT Mean 0.55 $30^{\text {th }}$ day Mean

0.45 $6^{\text {th }}$ day Mean

0.40
90 ${ }^{\text {th }}$ day Mean

0.40

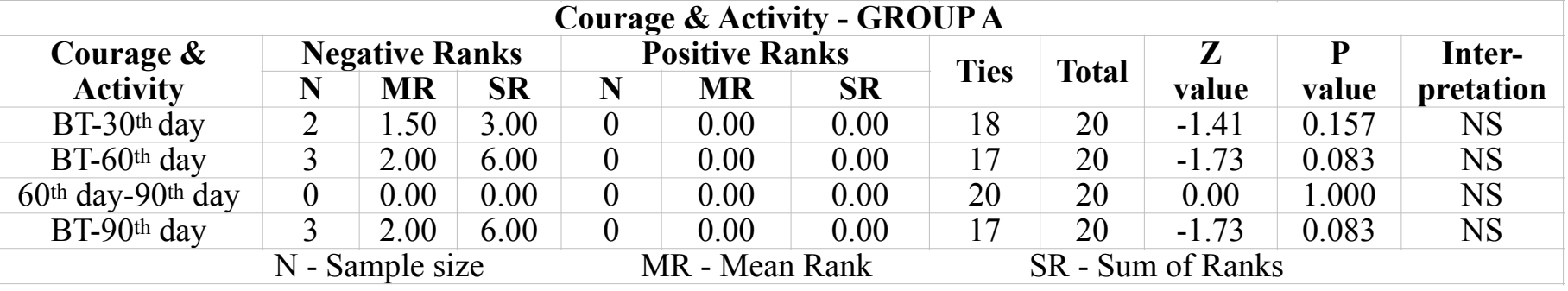

\section{Effect of treatment on Objective Parameters}

Statistical Analysis within the Group was interpreted by using Paired t test and in between Group A and Group B by Unpaired t test.

The mean score of Verbal IQ in Group A and Group B were 76.25, 76.99 before treatment and 78.13, 77.18 after treatment respectively. On follow up it was 78.73 and 77.18 respectively. There was $3.25 \%$ of improvement in Group A and $0.24 \%$ in Group B. The improvement before treatment \& after treatment and before treatment and on follow up in Group A was statistically highly significant with $p$ value $<0.001$ whereas statistically insignificant in Group B with $p$ value $>0.05$ on every level of assessment.

The mean score of Performance IQ in Group A and Group B were 77.51, 78.29 before treatment and 79.04, 78.39 after treatment respectively. On follow up it was 79.40 and 78.39 respectively. There was $2.43 \%$ of improvement in Group A and $0.12 \%$ in Group B. The improvement before treatment \& after treatment and before treatment and on follow up in Group A was statistically highly significant with $\mathrm{p}$ value $<0.001$ whereas statistically insignificant in Group B with p value $>0.05$ on every level of assessment.

The mean score of Verbal IQ in Group A and Group B were 76.88, 77.64 before treatment and 78.58, 77.78 after treatment respectively. On follow up it was 79.08 and 77.78 respectively. There was $2.86 \%$ of improvement in Group A and $0.18 \%$ in Group B. The improvement on before treatment \& on 30th day, before treatment \& after treatment and before treatment and on follow up in Group A was statistically highly significant with p value $<0.001$ whereas statistically insignificant in Group B with p value $>0.05$ on every level of assessment.

Table no.7: Results of Shankhapushpi Choorna for the parameter Verbal Scale VERBAL SCALE - GROUP A

\begin{tabular}{|c|c|c|c|c|c|c|c|c|}
\hline \multirow{2}{*}{$\begin{array}{l}\text { Verbal } \\
\text { Scale }\end{array}$} & \multirow{2}{*}{$\begin{array}{l}\text { Mean } \\
\text { Score }\end{array}$} & \multirow{2}{*}{$\begin{array}{l}\text { Difference } \\
\text { in Mean }\end{array}$} & \multicolumn{5}{|c|}{ Paired t Test } & \multirow[t]{2}{*}{ Interpretation } \\
\hline & & & $\%$ change & $\begin{array}{l}\text { Standard } \\
\text { Deviation }\end{array}$ & $\begin{array}{c}\text { Standard } \\
\text { Error Mean }\end{array}$ & t value & P value & \\
\hline BT & 76.25 & \multirow{2}{*}{-0.72} & \multirow{2}{*}{$0.94 \%$} & 3.09 & 0.69 & \multirow{2}{*}{-4.14} & \multirow{2}{*}{0.001} & \multirow{2}{*}{ HS } \\
\hline $30^{\text {th }}$ day & 76.97 & & & 3.22 & 0.72 & & & \\
\hline BT & 76.25 & \multirow{2}{*}{-1.88} & \multirow{2}{*}{$2.46 \%$} & 3.09 & 0.69 & \multirow{2}{*}{-6.81} & \multirow{2}{*}{0.000} & \multirow{2}{*}{ HS } \\
\hline $60^{\text {th }}$ day & 78.13 & & & 3.20 & 0.71 & & & \\
\hline $60^{\text {th }}$ day & 78.13 & \multirow{2}{*}{-0.60} & \multirow{2}{*}{$0.76 \%$} & 3.20 & 0.71 & \multirow{2}{*}{-3.52} & \multirow{2}{*}{0.002} & \multirow{2}{*}{$\mathrm{S}$} \\
\hline $90^{\text {th }}$ day & 78.73 & & & 3.22 & 0.72 & & & \\
\hline BT & 76.25 & \multirow{2}{*}{-2.48} & \multirow{2}{*}{$3.25 \%$} & 3.09 & 0.69 & \multirow{2}{*}{-6.86} & \multirow{2}{*}{0.000} & \multirow{2}{*}{ HS } \\
\hline $90^{\text {th }}$ day & 78.73 & & & 3.22 & 0.72 & & & \\
\hline
\end{tabular}


Table no.8: Results of Placebo for the parameter Verbal Scale

Verbal Scale - GROUP B

\begin{tabular}{|c|c|c|c|c|c|c|c|c|}
\hline \multirow[b]{2}{*}{$\begin{array}{l}\text { Verbal } \\
\text { Scale }\end{array}$} & \multirow[b]{2}{*}{$\begin{array}{l}\text { Mean } \\
\text { Score }\end{array}$} & \multirow[b]{2}{*}{$\begin{array}{l}\text { Difference } \\
\text { in Mean }\end{array}$} & \multicolumn{5}{|c|}{ Paired t Test } & \multirow[t]{2}{*}{ Interpretation } \\
\hline & & & $\%$ change & $\begin{array}{l}\text { Standard } \\
\text { Deviation }\end{array}$ & $\begin{array}{c}\text { Standard } \\
\text { Error Mean }\end{array}$ & t value & P value & \\
\hline $\begin{array}{c}\text { BT } \\
30^{\text {th }} \text { day }\end{array}$ & $\begin{array}{l}76.99 \\
77.15\end{array}$ & -0.16 & $0.20 \%$ & $\begin{array}{l}2.81 \\
2.95\end{array}$ & $\begin{array}{l}0.62 \\
0.66\end{array}$ & -1.50 & 0.148 & NS \\
\hline $\begin{array}{l}\text { BT } \\
60^{\text {th }} \text { day }\end{array}$ & $\begin{array}{l}76.99 \\
77.18\end{array}$ & -0.19 & $0.24 \%$ & $\begin{array}{l}2.81 \\
2.98\end{array}$ & $\begin{array}{l}0.62 \\
0.66\end{array}$ & -1.48 & 0.153 & NS \\
\hline $60^{\text {th }}$ day & 77.18 & & & 2.98 & 0.66 & & & \multirow{2}{*}{$\begin{array}{l}\text { Statistics } \\
\text { cannot be } \\
\text { computed }\end{array}$} \\
\hline $90^{\text {th }}$ day & 77.18 & & & 2.98 & 0.66 & & & \\
\hline $\begin{array}{c}\text { BT } \\
90^{\text {th }} \text { day }\end{array}$ & $\begin{array}{l}76.99 \\
77.18\end{array}$ & -0.19 & $0.24 \%$ & $\begin{array}{l}2.81 \\
2.98\end{array}$ & $\begin{array}{l}0.62 \\
0.66\end{array}$ & -1.69 & 0.153 & NS \\
\hline
\end{tabular}

Table no.9: Results of Shankhapushpi Choorna for the parameter Performance Scale Performance Scale - GROUP A

\begin{tabular}{|c|c|c|c|c|c|c|c|c|}
\hline \multirow[b]{2}{*}{$\begin{array}{l}\text { Performance } \\
\text { Scale }\end{array}$} & \multirow[b]{2}{*}{$\begin{array}{l}\text { Mean } \\
\text { Score }\end{array}$} & \multirow[b]{2}{*}{$\begin{array}{l}\text { Difference } \\
\text { in Mean }\end{array}$} & \multicolumn{5}{|c|}{ Paired t Test } & \multirow[t]{2}{*}{ Interpretation } \\
\hline & & & $\begin{array}{c}\% \\
\text { change }\end{array}$ & $\begin{array}{l}\text { Standard } \\
\text { Deviation }\end{array}$ & $\begin{array}{c}\text { Standard } \\
\text { Error } \\
\text { Mean }\end{array}$ & t value & P value & \\
\hline BT & 77.51 & \multirow{2}{*}{-0.48} & \multirow{2}{*}{$0.60 \%$} & 3.99 & 0.89 & \multirow{2}{*}{-2.79} & \multirow{2}{*}{0.012} & \multirow{2}{*}{$\mathrm{S}$} \\
\hline $30^{\text {th }}$ day & 77.99 & & & 3.96 & 0.88 & & & \\
\hline BT & 77.51 & \multirow{2}{*}{-1.53} & \multirow{2}{*}{$1.97 \%$} & 3.99 & 0.89 & \multirow{2}{*}{-4.47} & \multirow{2}{*}{0.000} & \multirow{2}{*}{ HS } \\
\hline $60^{\text {th }}$ day & 79.04 & & & 3.96 & 0.88 & & & \\
\hline $60^{\text {th }}$ day & 79.04 & \multirow{2}{*}{-0.36} & \multirow{2}{*}{$0.45 \%$} & 3.96 & 0.88 & \multirow{2}{*}{-1.86} & \multirow{2}{*}{0.077} & \multirow{2}{*}{ NS } \\
\hline $90^{\text {th }}$ dav & 79.40 & & & 4.02 & 0.90 & & & \\
\hline BT & 77.51 & \multirow{2}{*}{-1.89} & \multirow{2}{*}{$2.43 \%$} & 3.99 & 0.89 & \multirow{2}{*}{-5.55} & \multirow{2}{*}{0.000} & \multirow{2}{*}{ HS } \\
\hline $90^{\text {th }}$ day & 79.40 & & & 4.02 & 0.90 & & & \\
\hline
\end{tabular}

Table no.10: Results of Placebo for the parameter Performance Scale PERFORMANCE SCALE - GROUP B

\begin{tabular}{|c|c|c|c|c|c|c|c|c|}
\hline \multirow{3}{*}{$\begin{array}{l}\text { Performance } \\
\text { Scale }\end{array}$} & \multirow{3}{*}{$\begin{array}{l}\text { Mean } \\
\text { Score }\end{array}$} & \multirow{3}{*}{$\begin{array}{l}\text { Difference } \\
\text { in Mean }\end{array}$} & \multirow{2}{*}{\multicolumn{5}{|c|}{ Paired t Test }} & \multirow{3}{*}{ Interpretation } \\
\hline & & & & & & & & \\
\hline & & & $\%$ change & $\begin{array}{l}\text { Standard } \\
\text { Deviation }\end{array}$ & $\begin{array}{c}\text { Standard } \\
\text { Error Mean }\end{array}$ & t value & P value & \\
\hline BT & 78.29 & \multirow{2}{*}{-0.10} & \multirow{2}{*}{$0.12 \%$} & 2.93 & 0.65 & \multirow{2}{*}{-1.69} & \multirow{2}{*}{0.106} & \multirow{2}{*}{ NS } \\
\hline $\begin{array}{c}30^{\text {th }} \text { day } \\
\text { BT }\end{array}$ & 78.39 & & & 2.91 & 0.65 & & & \\
\hline $\begin{array}{c}\text { BT } \\
60^{\text {th }} \text { day }\end{array}$ & $\begin{array}{l}78.29 \\
7830\end{array}$ & -0.10 & $0.12 \%$ & 2.93 & 0.65 & -1.69 & 0.106 & NS \\
\hline $60^{\text {th }}$ day & 78.39 & & \multirow[b]{2}{*}{ - } & 2.91 & 0.65 & \multirow[b]{2}{*}{ - } & \multirow[b]{2}{*}{ - } & \multirow{2}{*}{$\begin{array}{c}\text { Statistics cannot } \\
\text { be computed }\end{array}$} \\
\hline $90^{\text {th }}$ day & 78.39 & & & 2.91 & 0.65 & & & \\
\hline BT & 78.29 & \multirow{2}{*}{-0.10} & \multirow{2}{*}{$0.12 \%$} & 2.93 & 0.65 & \multirow{2}{*}{-1.69} & \multirow{2}{*}{0.106} & \multirow{2}{*}{ NS } \\
\hline $90^{\text {th }}$ day & 78.39 & & & 2.91 & 0.65 & & & \\
\hline
\end{tabular}

Table no.11: Results of Shankhapushpi Choorna for the parameter Full Scale IQ FULL SCALE IQ - GROUP A

\begin{tabular}{|c|c|c|c|c|c|c|c|c|}
\hline \multirow{3}{*}{$\begin{array}{c}\text { Full Scale } \\
\text { IQ }\end{array}$} & \multirow{3}{*}{$\begin{array}{l}\text { Mean } \\
\text { Score }\end{array}$} & \multirow{3}{*}{$\begin{array}{l}\text { Difference } \\
\text { in Mean }\end{array}$} & \multicolumn{5}{|c|}{ FULL SCALE IQ - GROUP A } & \multirow{3}{*}{ Interpretation } \\
\hline & & & \multicolumn{5}{|c|}{ Paired T Test } & \\
\hline & & & $\%$ change & $\begin{array}{l}\text { Standard } \\
\text { Deviation }\end{array}$ & $\begin{array}{c}\text { Standard } \\
\text { Error Mean }\end{array}$ & t value & Pvalue & \\
\hline BT & 76.88 & \multirow{2}{*}{-0.60} & \multirow{2}{*}{$0.78 \%$} & 3.40 & 0.76 & \multirow{2}{*}{-5.9} & \multirow{2}{*}{0.000} & \multirow{2}{*}{ HS } \\
\hline $30^{\text {th }}$ day & 77.48 & & & 3.42 & 0.76 & & & \\
\hline BT & 76.88 & \multirow{2}{*}{-1.70} & \multirow{2}{*}{$2.21 \%$} & 3.40 & 0.76 & \multirow{2}{*}{-8.78} & \multirow{2}{*}{0.000} & \multirow{2}{*}{ HS } \\
\hline $60^{\text {th }}$ day & 78.58 & & & 3.37 & 0.75 & & & \\
\hline $60^{\text {th }}$ day & 78.58 & \multirow{2}{*}{-0.49} & \multirow{2}{*}{$0.62 \%$} & 3.37 & 0.75 & \multirow{2}{*}{-3.25} & \multirow{2}{*}{0.004} & \multirow{2}{*}{$\mathrm{S}$} \\
\hline $90^{\text {th }}$ day & 79.08 & & & 3.40 & 0.76 & & & \\
\hline BT & 76.88 & \multirow{2}{*}{-2.20} & \multirow{2}{*}{$2.86 \%$} & 3.40 & 0.76 & \multirow{2}{*}{-9.04} & \multirow{2}{*}{0.000} & \multirow{2}{*}{ HS } \\
\hline $90^{\text {th }}$ day & 79.08 & & & 3.40 & 0.76 & & & \\
\hline
\end{tabular}


Table no.12: Results of Placebo for the parameter Full Scale IQ

FULL SCALE IQ - GROUP B

\begin{tabular}{|c|c|c|c|c|c|c|c|c|}
\hline \multirow{3}{*}{$\begin{array}{c}\text { Full } \\
\text { Scale IQ }\end{array}$} & \multirow[b]{3}{*}{$\begin{array}{l}\text { Mean } \\
\text { Score }\end{array}$} & \multirow[b]{3}{*}{$\begin{array}{l}\text { Difference } \\
\text { in Mean }\end{array}$} & \multirow{2}{*}{\multicolumn{5}{|c|}{ Paired t Test }} & \multirow{3}{*}{ Interpretation } \\
\hline & & & & & & & & \\
\hline & & & $\%$ change & $\begin{array}{l}\text { Standard } \\
\text { Deviation }\end{array}$ & $\begin{array}{c}\text { Standard } \\
\text { Error Mean }\end{array}$ & t value & P value & \\
\hline BT & 77.64 & \multirow{2}{*}{-0.13} & \multirow{2}{*}{$0.16 \%$} & 2.66 & 0.59 & \multirow{2}{*}{-2.02} & \multirow{2}{*}{0.057} & \multirow{2}{*}{ NS } \\
\hline $30^{\text {th }}$ day & 77.77 & & & 2.67 & 0.59 & & & \\
\hline BT & 77.64 & \multirow{2}{*}{-0.14} & \multirow{2}{*}{$0.18 \%$} & 2.66 & 0.59 & \multirow{2}{*}{-1.96} & \multirow{2}{*}{0.064} & \multirow{2}{*}{ NS } \\
\hline $60^{\text {th }}$ day & 77.78 & & & 2.67 & 0.59 & & & \\
\hline $60^{\text {th }}$ day & 77.78 & & \multirow[b]{2}{*}{. } & 2.67 & 0.59 & \multirow[b]{2}{*}{-} & & \multirow{2}{*}{$\begin{array}{c}\text { Statistics cannot } \\
\text { be computed }\end{array}$} \\
\hline $90^{\text {th }}$ day & 77.78 & & & 2.67 & 0.59 & & & \\
\hline BT & 77.64 & \multirow{2}{*}{-0.14} & \multirow{2}{*}{$0.18 \%$} & 2.66 & 0.59 & \multirow{2}{*}{-1.96} & \multirow{2}{*}{0.064} & \multirow{2}{*}{ NS } \\
\hline $90^{\text {th }}$ day & 77.78 & & & 2.67 & 0.59 & & & \\
\hline
\end{tabular}

Comparison between the groups for subjective \& objective parameters implied that Group A showed better results with $\mathrm{p}$ value $<0.001$ which is statistically highly significant. Hence Shankhapushpi Choorna proved its efficacy on improvement of borderline IQ.

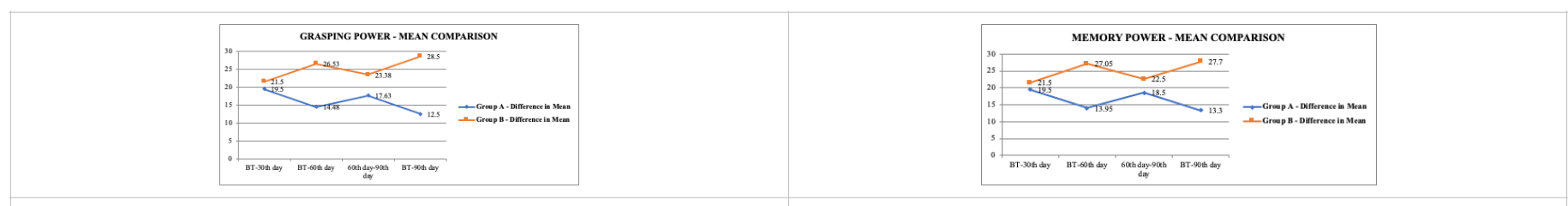

Graph No.1: Showing comparative effect on Grasping Power in Group A \& B
Graph No.2: Showing comparative effect on Memory Power in Group A \& B

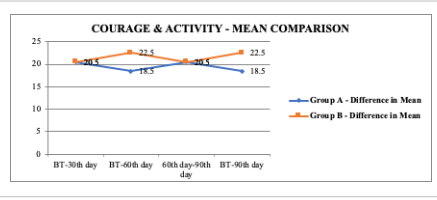

Graph No.3: Showing comparative effect on Courage \& Activity in Group A \& B
Graph No.4: Showing comparative effect on Verbal IQ Score in Group A \& B
Graph No.5: Showing comparative effect on Performance IQ Score in Group A and B

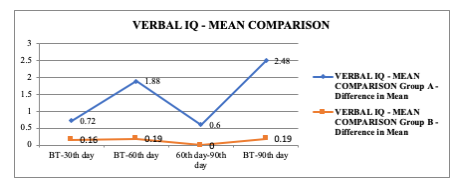

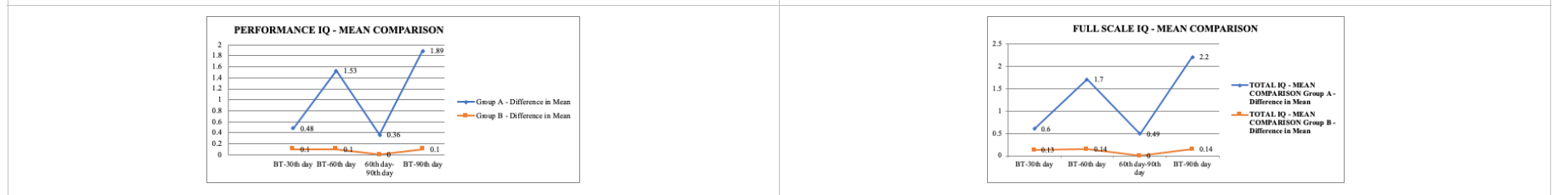

\section{Graph No.6: Showing comparative effect on Full Scale IQ Score in Group A \& B}

\section{Discussion}

The time validated documentation of the eternal concepts of Ayurveda which explores the practical application is vital for the conservation of health of the present era. Intellectual assessment and intelligence testing are an approach that gives an idea of individual's general intellectual functioning and cognitive abilities. General intellectual functioning typically assigns to one's global or overall level of intelligence, often referred to as Intelligence Quotient (IQ) (13). The typical feature of borderline IQ is that they exhibit less positive and less sensitive behaviour as compared to peers. But they are less behaviourally troublesome than that of the children with other behavioural problems. They can be considered as the vulnerable group whom needs special interventions and it will definitely make improvement in their demanding issues (14).

IQ tests are sensitive to a number of cognitive difficulties and answers a number of questions about the distinctive nature of children of the same age. It is a powerful standard of the integrity of the individual child's problem - solving system. The review of intellectual strengths and weaknesses provides important information about the child's specific educational needs. When IQ tests are used with other cognitive measures, a clinician is able to obtain key information about the child's learning style resulting in 
appropriate recommendations for educational intervention, modification or accommodation (15). Malin's Intelligence Scale for Indian Children (MISIC) is the Indian adaptation of Wechsler's Intelligence Scale for Children is used to assess the IQ of the children that provide scores on Verbal IQ, Performance IQ and FullScale IQ (16). MISIC includes tests based on Information, Comprehension, Arithmetic, Similarities, Vocabulary, Digit Span, Picture Completion, Block Design, Object Assembly, Coding and Mazes is the ideal tool for assessment of each and every aspect of IQ of children.

Acharya Charaka defines Buddhi as the one which determines the specific qualities of the objects and impels the individual to speak or act intelligently. Buddhi is the matter responsible for conclusive knowledge. According to Chakrapani, Buddhi gives an initiation to work and to come to final conclusion after proper analysis (17). The key instruments of knowledge are Manas, Buddhi and Indriyas. Their interrelation with the empirical soul results in Karma, Vedana and understanding. Without this association nothing exists accordingly (18).

The critical analysis of the unique concept of Medha is only possible if the available scattered references are well organized. Medha is a faculty of Buddhi that can be interpreted as a specific psychic ability which is responsible for encoding and retaining a large amount of knowledge. Though the terms Dhee, Buddhi etc. are used synonymously to Medha, practically these are the separate entities having individual role. Buddhi, Medha, Dhee, Dhruti and Smruti can be considered as the peculiar aspects of human intellectual entities. The term Medhya in Ayurveda is described in a comprehensive way. It comprises of all the three mental faculties - Dhee, Dhruti and Smruti those are interrelated with each other in their functional aspects. Medhya can also be fractionalized into Grahanashakti (Power of Grasping), Dharanashakti (Power of Retention), Vivekashakti (Power of Discrimination) and Smruti (Power of Recollection) (19). Medhya Rasayanas are a group of medicinal plants with multi benefits specially to improve memory and intellect by its Prabhava (specific action). These drugs are known to have determined effects on mental performance by enhancing the functions of Buddhi and Manas.

As per Acharya Charaka, Rasayana is defined as the means of achieving the finest quality of Rasadi Dhatus where it promotes life span, improves Medha, cures diseases, stabilizes youthfulness, improves lustre, complexion, voice and makes body and senses strong and healthy (20). Rasayana drugs generally serves as Immunomodulator, Adaptogen, Antioxidant and Nootropic measures. Nootropic drugs will promote intellectual functions and brain integrity points towards the Medhya Rasayanas. Medhya drugs having the capacity to act at different levels in our body; at the level of Rasa, acts by stimulating and improving the functions of Agni, improves circulation of Rasa by Srotosodhana guna thus improving Medhya function.

Guru Snigdha Picchila Saraguna along with Seetavirya and Madhuravipaka of Shankhapushpi promotes Tarpaka \& Avalambaka Kapha thus enhances Dharanakarma and does Mastishkaposhana improving the power of retention. Acharya Vagbhata highlighting the Medhya action of Tiktarasa which is the pradhanarasa of Shankhapushpi (21). Shankhapushpi being Tikta Katu rasa predominance, promotes Pitta and enhances Grahana and Smarana (Grasping Power \& Memory). The Deepana, Pachana and Srotosodhana guna of the drug will enhances the Pachaka, Sadhaka and Alochaka Pitta qualities and act at the level of Manovaha and Rasavaha Srotas thus regulating its normal functioning. Since all the Tridoshas are involving in the vitiation of normal functioning of the Manas, drug with Tridoshasamaka property is necessary for the demand. Shankhapushpi by its Tridoshasamaka properties and Vata (Regulator of Manas) controlling activity, helps to normalize the involvement of Manodosha (Rajas \& Tamas). The factors Cintya, Vicharya, Uhya, Dhyeya and Sankalpya, emphasizes the whole memory process will regulated by the Medhya and Tridoshasamaka properties of Shankhapushpi.

Nootropics are the pharmacological group referred to as Brain tonics, Memory enhancers and Cognitive boosters. Nootropics are thought to work by altering the availability of supply of neurochemicals (neurotransmitters, enzymes and hormones) to the brain, by improving the brain's oxygen supply or by stimulating nerve growth that helps to enrich the functional aspects of the brain. Several types of chemical constituents like Alkaloids, Terpenoids, Phenolics, Flavonoids and Coumarins are reported scientifically for the pharmacodynamics of intellect and memory boosting action of the drug Shankhapushpi. Different studies concluded the neuroprotective activity of the drug is by lowering Beta-Amyloid deposition in the brain offers protection from memory dysfunction. Also, the underlying mechanism considering free radical scavenging and antioxidant action on the brain cells, will provide protective action against brain aging. Shankhapushpi is being an AchE inhibitor, which protect the loss of neurotransmitter AchE, directly responsible for the cognitive functions (22).

\section{Conclusion}

Over the duration of the study, the trial drug Shankhapushpi Choorna was seen to have a positive effect on all the subjective and objective parameters with statistically highly significant results. Hence it can be concluded that Shankhapushpi Choorna with the proper dosage according to the age definitely improves the Medha of children with borderline intellectual functioning. The drug can be used as both promotive as well as curative aspects in accordance with intelligence. 


\section{References}

1. Sreekumar T. Astanga Hrdaya of Vagbhata. 1st edition. Thrissur; Publication department, Harisree Hospital; 2008. p.25.

2. Gayle A. Brazeau. Is There Time for Student Intellectual Development and Scholarly Pursuits. Am J Pharm Educ. March, 2010; 74(2); p.18.

3. https://www.researchgate.net/IQ (The Intelligence Quotient). dated 04-02-2020 time 16:30 IST.

4. Sharma R.K \& Bhagwan Dash. Caraka Samhita with Cakrapani Datta's Ayurveda Dipika. 1 $1^{\text {st }}$ edition. Varanasi; Chaukhamba Sanskrit Series office; 2010. p.277.

5. Pramod Shukla Shailendra, Chitrakar. M. Efficacy of Jyothishmati Oil with Pranayama on IQ. International Journal of Ayurveda and Pharma Research. August, 2015; 3(8); 100.

6. The Best Test Preparation for the Advanced Placement Examination in Psychology, Research \& Education Association. 2003, p.99.

7. Sharma R.K \& Bhagwan Dash. Caraka Samhita with Cakrapani Datta's Ayurveda Dipika. 1 ${ }^{\text {st }}$ edition. Varanasi; Chaukhamba Sanskrit Series office; 2010. p.336-338.

8. Sharma R.K \& Bhagwan Dash. Caraka Samhita with Cakrapani Datta's Ayurveda Dipika. 1 $1^{\text {st }}$ edition. Varanasi; Chaukhamba Sanskrit Series office; 2010. p.166.

9. Bhanuji Diksita. Sastri Haragovinda. Namalinganusasana or Amarakosha of Amarasimha. $1^{\text {st }}$ edition. Varanasi; Chaukhambha Sanskrit Sansthan; 2016. p.72.

10. Byadgi S. Parameswarappa, Pandey Kumar Ajai. A textbook of Kayacikitsa. $1^{\text {st }}$ edition. Varanasi; Chaukhambha Sanskrit Sansthan; 2018. p.916.

11. Sharma R.K \& Bhagwan Dash. Caraka Samhita with Cakrapani Datta's Ayurveda Dipika. 1 $1^{\text {st }}$ edition. Varanasi; Chaukhamba Sanskrit Series office; 2010. p.46.
12. Ayurvedic Pharmacopoeia of India. Part 1, Vol 2; p.147.

13. Ortiz O. Samuel, Lella A. Stacey. Intellectual ability and assessment: A primer for parents and educators. NASP. 2010; P.S2H12/1-4.

14. Wieland Jannelien, Zitman G. Frans. It is time to bring borderline intellectual functioning back into the main fold of classification systems. BJPsych Bull. August, 2016; 40(4); p.204-206.

15. Holdnack A. James. Defining the role of intellectual and cognitive assessment in special education. The Psychological Corporation, a Harcourt Assessment Company; Psychology. 2003; p.12.

16. Malin A. J. Manual of Malin's Intelligence Scale for Indian Children. Lucknow: Indian Psychological Corporation. Chapter 2, General Description of the WISC and MISIC; p.3.

17. Sharma R.K \& Bhagwan Dash. Caraka Samhita with Cakrapani Datta's Ayurveda Dipika. $1^{\text {st }}$ edition. Varanasi; Chaukhamba Sanskrit Series office; 2010. p.317.

18. Sharma R.K \& Bhagwan Dash. Caraka Samhita with Cakrapani Datta's Ayurveda Dipika. 1 ${ }^{\text {st }}$ edition. Varanasi; Chaukhamba Sanskrit Series office; 2010. p.325.

19. Ray Suman. Ray Asim. Medhya Rasayanas in Brain function and Disease. Medicinal Chemistry. 2015; 5(12); p.505-506.

20. Sharma R.K \& Bhagwan Dash. Caraka Samhita with Cakrapani Datta's Ayurveda Dipika. 1st edition. Varanasi; Chaukhamba Sanskrit Series office; 2010. p.8.

21. Sreekumar T. Astanga Hrdaya of Vagbhata. 1 ${ }^{\text {st }}$ edition. Thrissur; Publication department, Harisree Hospital; 2008. p. 275.

22. Sethiya N. K. Mishra S. H. Neuropharmacological evaluation on four traditional herbs used as nervine tonic and commonly available as Shankhapushpi in India. Journal of Ayurveda and Integrative Medicine. January-March, 2019; 10(1); p.25-31. 